\title{
DOLCETA, EDUCACIÓN ONLINE PARA LOS CONSUMIDORES: MÓDULO DE ALFABETIZACIÓN FINANCIERA EN ESPAÑA
}

\author{
María-Victoria Sánchez-Rebull, Fernando Campa-Planas y Ana B. Hernández-Lara
}

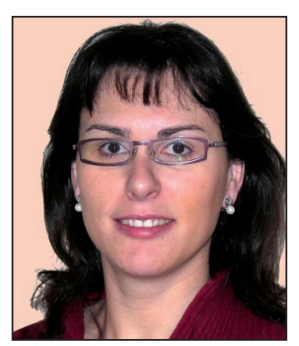

María-Victoria Sánchez-Rebull es doctora en gestión de empresas por la Universitat Rovira i Virgili (URV) y está vinculada desde 1991 al Área de Economía Financiera y Contabilidad del Departamento de Gestión de Empresas de la URV del cual actualmente es directora. Ha sido coordinadora del máster de contabilidad y auditoría de cuentas, del máster de gestión de empresas turísticas de la URV, y responsable académica del máster oficial en dirección y planificación de turismo y ocio, de la misma universidad. Es autora de publicaciones y artículos, así como de comunicaciones en congresos nacionales e internacionales sobre contabilidad de gestión adaptada al sector turístico y la docencia universitaria y el EEES.

Universidad Rovira i Virgili Departamento de Gestión de Empresas Facultad de Ciencias Económicas y Empresariales Av. Universitat, 1. Reus (Tarragona), España mariavictoria.sanchez@urv.cat

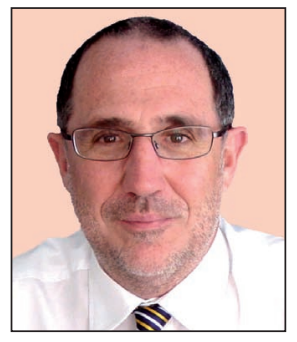

Fernando Campa-Planas es licenciado en ADE por la Universitat Politècnica de Catalunya (UPC), MBA por Esade y doctor por la Universidad Rovira i Virgili (URV). Inició su perfil financiero en la auditora Arthur Andersen, prosiguiendo su carrera como directivo en el Grupo INI, director de control y seguimiento en Iberia y posteriormente director de administración y finanzas en Port Aventura. Desde 2005 es docente en la URV y tiene diversas publicaciones y comunicaciones vinculadas al sector hotelero, parques temáticos, compañías aéreas y, en general, de gestión vinculada al sector turístico y a la innovación docente.

Universidad Rovira i Virgili Departamento de Gestión de Empresas Facultad de Ciencias Económicas y Empresariales Av. Universitat, 1. Reus (Tarragona), España fernando.campa@urv.cat

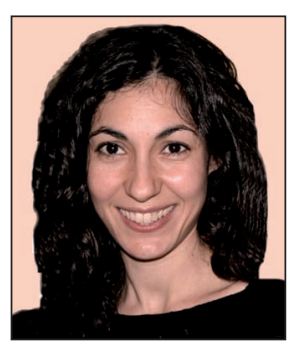

Ana B. Hernández-Lara, doctora en organización de empresas por la Universidad Pablo de Olavide, Sevilla, en 2007, es profesora agregada en el Área de Organización de Empresas del Departamento de Gestión de Empresas de la Universidad Rovira i Virgili, donde trabaja desde 2007. Es autora de diversas publicaciones nacionales e internacionales en revistas indexadas en los $J C R$, y ha presentado comunicaciones en congresos nacionales e internacionales de prestigio sobre dirección estratégica, gobierno corporativo e innovación, así como en otros relacionados con la innovación y la calidad docente.

Universidad Rovira i Virgili Departamento de Gestión de Empresas Facultad de Ciencias Económicas y Empresariales Av. Universitat, 1. Reus (Tarragona), España anabeatriz.hernandez@urv.cat

\section{Resumen}

Dolceta (Development of online consumer education tool for adults) es un proyecto educativo online impulsado por la Comisión Europea que implica a 28 países de la misma, y pretende desarrollar la educación para el consumo basándose en las nuevas tecnologías de la información. Se describe el módulo sobre alfabetización financiera que se elaboró en el caso de la plataforma española. Se explica cómo se tomaron las decisiones relacionadas con este módulo, y la coordinación que tuvo lugar con los equipos de los países participantes para construir contenidos y actividades. Se expone cómo se organizó y llevó a cabo esta experiencia adaptada al contexto español, para que pueda ser utilizada en otros países y/o entornos y pueda contribuir a la difusión y homogeneidad de una mejor alfabetización financiera. Con esta herramienta se remarca la necesidad de estas experiencias formativas en la sociedad, especialmente desde edades tempranas.

\section{Palabras clave}

Educación del consumidor, Alfabetización financiera, Aprendizaje electrónico, Proyecto educativo europeo.

\section{Title: Dolceta online consumer education: development of the financial education module in Spain}




\begin{abstract}
Dolceta (Development of online consumer education tool for adults) is an ongoing project involving 28 countries of the European Union, with support from the European Commission. The main objective of this paper is to describe how the Dolceta module on financial education was constructed in the case of the Spanish platform, how decisions related to this module were made, and the cooperation and coordination processes developed to construct the contents and activities of this module in line with the other participating European countries. Thus, we intend to describe the organization and development at the European level of a learning experience through the use of new information and communication technologies (ICT) in the fields of financial and consumer education, in order to publicize this experience and to guide other similar initiatives, especially at an early stage.
\end{abstract}

\title{
Keywords
}

Consumer education, financial education, e-learning, European educational project.

Sánchez-Rebull, María-Victoria; Campa-Planas, Fernando; Hernández-Lara, Ana B. "Dolceta, educación online para los consumidores: módulo de alfabetización financiera en España". El profesional de la información, 2011, noviembrediciembre, v. 20, n. 6, pp. 682-688

http://dx.doi.org/10.3145/epi.2011.nov.13

\section{Introducción}

Dolceta es un proyecto de educación online que pretende educar para el consumo en el que participan 28 países de la Unión Europea. Está promovido y financiado por la Dirección General de Sanidad y Consumo (DG Sanco) de la UE, y está llevado a cabo técnicamente por la European University Continuing Education Network (Eucen). Ha producido módulos online sobre diferentes temas de consumo: derechos de los consumidores, servicios, etc.

Por un lado trata de ofrecer a los consumidores información útil para la vida diaria y les alienta a utilizarla para tomar decisiones seguras de compra. Por otro, proporciona contenidos y herramientas para mejorar la educación de los estudiantes para un consumo más responsable. Es decir, trata de educar a la gente y mejorar su comportamiento en términos de consumo responsable.

El propósito es importante dada la creciente necesidad de educación de los consumidores a escala europea (Bailey; Kitson, 2006; Ministros europeos de educación, 1999), y puede extrapolarse a otros ámbitos. Los consumidores necesitan formación que pueda ayudarles a gestionar situaciones de consumo complejas, a las que se enfrentan cotidianamente (Arvanitis, 2010; Carnell; Lodge, 2002; Stoll; Fink; Earl, 2004).

Esto sucede especialmente en el mundo económico y financiero, mucho más desconocido, en el que adicionalmente los consumidores deben afrontar la alfabetización financiera como una experiencia de formación continua (Alrø; Skovsmose; Skanstrøm, 2000; Bailey; Kitson, 2006; Gottschau; Nielsen, 2005) que debe iniciarse cuanto antes. Es importante formar a los profesores y proveerles de las herramientas necesarias para fomentar la calidad de dicha alfabetización, desde edades tempranas.

Esta necesidad formativa no afecta a un solo país, estamento social o grupo de edad, sino que resulta importante para toda la Unión Europea. Expertos reunidos en el Segundo Encuentro Europeo de Expertos sobre Educación Financiera celebrado en Bruselas en 2007 concluyeron que a pesar de haber provocado muchos problemas, la crisis financiera ha abierto una oportunidad para promover la educación financiera y facilitarla a los ciudadanos. Para ello resultaba útil intercambiar experiencias y colaborar entre los Estados miembros, lo que podría extrapolarse a otros países de fuera de la UE.

http://www. financialcapability.ie/index.jsp? $p=585 \& n=591$

Un objetivo resaltable de Dolceta es alcanzar una alfabetización financiera homogénea, pero adaptada al contexto y peculiaridades de cada país, utilizando las TIC, cuyo espectacular desarrollo está transformando radicalmente la enseñanza y el aprendizaje (Ma; Vogel; Wagner, 2000). Las TIC permiten acceder a la educación a más personas, ofreciéndoles nuevas formas de aprender y estudiar, y provocando la aparición de nuevas herramientas, materiales y actividades online que facilitan el aprendizaje y la interacción entre profesores y alumnos, sin restricciones de tiempo o espacio (Barker, 2002; Benito, 2009; Cabero; Gisbert, 2005; Sun et al., 2008; McGill; Klobas, 2009).

Sin embargo sorprende que la bibliografía y la investigación sobre alfabetización financiera sea escasa. De hecho son pocas las investigaciones publicadas en revistas internacionales académicas. La mayoría describe experiencias específicas de enseñanza financiera online, o casos concretos en los que se ha aplicado alguna herramienta de e-learning (juegos de negocios, etc.) en temas de finanzas o consumo (Robinson, 2008; Yamashita; Takahashi; Terano, 2008).

Este artículo tiene estos propósitos:

- describir el proceso de construcción del módulo de alfabetización financiera de la plataforma educativa Dolceta en el contexto español, cómo se tomaron las decisiones, y los procesos de cooperación y coordinación establecidos entre los distintos países europeos participantes.

- describir la organización y desarrollo de esta experiencia de e-learning, con el fin de darla a conocer y servir de orientación a otras iniciativas similares, contribuyendo así a la escasa literatura sobre e-learning en el ámbito financiero. 
- resaltar la importancia de promover la alfabetización financiera para apoyar las decisiones cotidianas, ofrecer a los profesores una herramienta de calidad y homogénea a escala europea.

\section{Contenidos y estructura del módulo de alfabetización financiera de Dolceta}

Dolceta ofrece varios módulos formativos interactivos que incluyen recursos didácticos sobre los derechos del consumidor, los servicios vinculados al consumo y el consumo responsable. Son de dos tipos: orientados a profesores (para su uso como recurso didáctico en el aula); y dirigidos directamente a los consumidores y/o estudiantes. En agosto de 2011 los módulos implantados en la plataforma española eran ocho (figura 1):

\section{Para profesores}

- Educación para el consumo

- Alfabetización financiera

- Consumo sostenible

\section{Para estudiantes y consumidores}

- Derechos del consumidor

- Servicios (energía, telecomunicaciones, transportes, etc.)

- Seguridad de los productos

- Consumo sostenible

- Servicios financieros

\subsection{Módulo de alfabetización financiera}

Permite a consumidores y a estudiantes:

- Encontrar información para llevar a cabo operaciones financieras simples o relativamente complejas.

- Ser capaz de identificar sus necesidades en relación a asuntos financieros.

- Comprender los principios básicos sobre cómo financiar sus necesidades de compra.

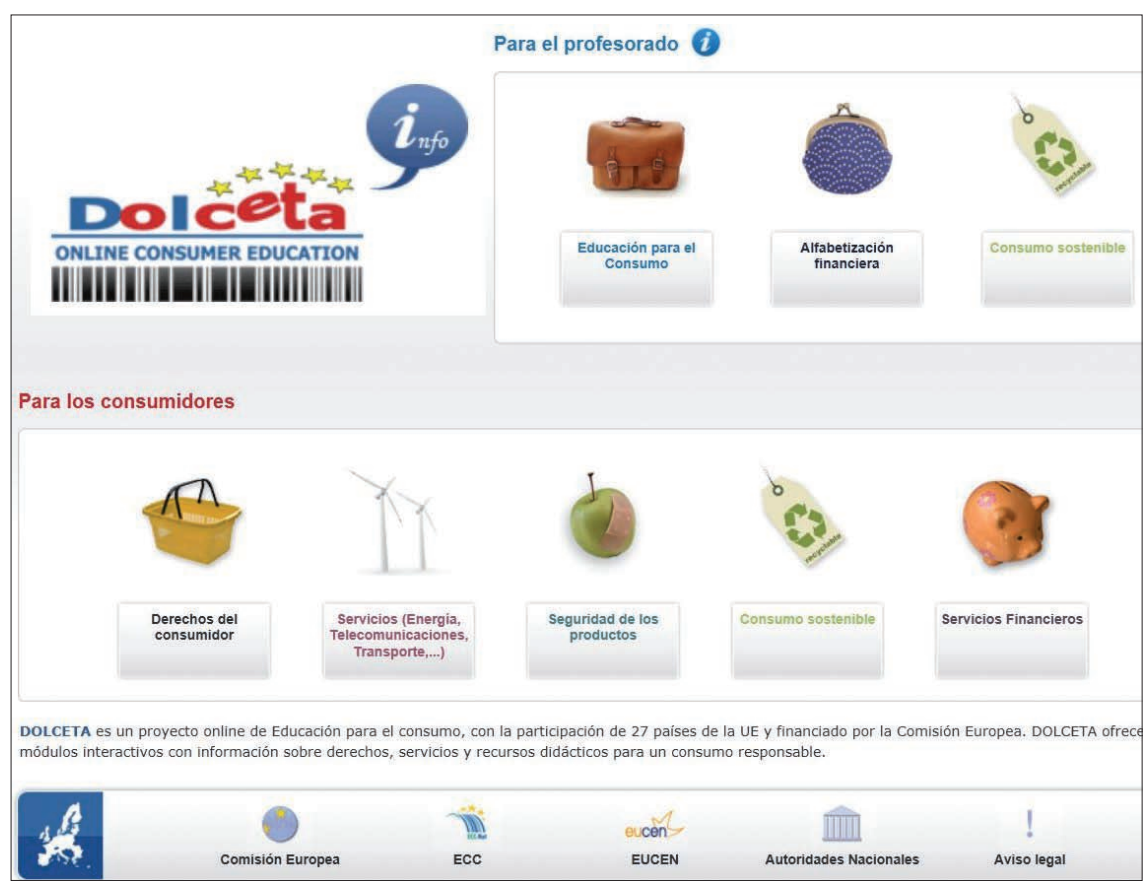

Figura 1. Módulos en la plataforma española de Dolceta, http://www.dolceta.eu/espana
- Entender la necesidad de obtener información y comparar las ofertas recibidas antes de iniciar una compra.

- Prever consecuencias de las decisiones adoptadas y, en particular, los riesgos derivados de los contratos (no leer el clausulado, no entender sus consecuencias, etc.).

- Tomar conciencia de su responsabilidad en las decisiones financieras que adopten.

Estas habilidades y responsabilidades varían según la edad del estudiante o usuario. Por ello se establecieron tres niveles: estudiantes de primaria, de secundaria y de bachillerato. Es preciso resaltar que el material publicado no trata de reemplazar el asesoramiento especializado de profesionales, sino constituir una herramienta adicional de guía e información.

\subsection{Materias financieras y lecciones del módulo}

Se incluye una introducción, cuatro secciones, un glosario y una serie de fichas técnicas.

\section{Introducción}

La introducción da una respuesta precisa a tres cuestiones clave: ¿Por qué enseñar alfabetización financiera? ¿Cómo enseñar alfabetización financiera? y ¿Qué enseñar en alfabetización financiera? Es un apartado considerado obligatorio para todos los países y, a partir de una propuesta, debía redactarse y adaptarse a cada contexto específico.

\section{Secciones}

Son: Gastos, Ahorro e inversión, Préstamos y créditos, y Seguros y protección.

Cada sección se enfoca a tres niveles de conocimiento: básico, intermedio y avanzado, correspondientes a diferentes franjas de edad de los estudiantes (hasta 10, 15 y 18 años, dependiendo de las lecciones, respectivamente).

Cada sección está dividida en 6 lecciones o temáticas, que fueron elegidas por cada país a partir de una propuesta de un panel de expertos. Así, por ejemplo, la lección sobre apuestas no se ha implementado en España por no considerarla apropiada y evitar así la propensión al juego.

En la plataforma española, las 6 lecciones planteadas para la cuarta sección "Seguros y protección" se muestran en la tabla 1.

Cada lección (tabla 1 ) está dirigida a diferentes niveles de edad (dos a primaria, dos a secundaria y dos a estudiantes de bachillerato. Además, cada lección debe contener una descripción (tabla 2) y una estructura de contenidos establecidos (tabla 3).

La lección "¿Podemos cuantificar el riesgo?", por ejemplo, se ha planteado para 2 clases de 60 minutos. La principal competencia a enseñar es conocer las principales situaciones de riesgo que nos podemos encontrar, y tratar 


\begin{tabular}{|l|c|c|c|}
\hline & Primaria & $\begin{array}{c}\text { Secun- } \\
\text { daria }\end{array}$ & $\begin{array}{c}\text { Bachi- } \\
\text { llerato }\end{array}$ \\
\hline $\begin{array}{l}\text { ¿Estamos seguros? ¿Qué pode- } \\
\text { mos hacer para estarlo? }\end{array}$ & $\mathrm{x}$ & & \\
\hline Tipos de seguros & $\mathrm{x}$ & & \\
\hline $\begin{array}{l}\text { Cuando las cosas van mal, } \\
\text { ¿cómo ponemos una denuncia? }\end{array}$ & & $\mathrm{x}$ & \\
\hline $\begin{array}{l}\text { ¿Qué hacemos si una compra no } \\
\text { nos ha salido bien? }\end{array}$ & & $\mathrm{x}$ & \\
\hline Principales seguros & & & $\mathrm{x}$ \\
\hline ¿Podemos cuantificar el riesgo? & & & $\mathrm{x}$ \\
\hline
\end{tabular}

Tabla 1. Lecciones de la sección 4, "Seguros y protección"

\begin{tabular}{|l|l|}
\hline \multicolumn{1}{|c|}{ Título } & \multicolumn{1}{c|}{ ¿Podemos cuantificar el riesgo? } \\
\hline Ámbito & Economía, finanzas, matemáticas \\
\hline $\begin{array}{l}\text { Grupo de } \\
\text { edad }\end{array}$ & 16-18 años; bachillerato \\
\hline $\begin{array}{l}\text { Breve } \\
\text { descripción }\end{array}$ & $\begin{array}{l}\text { Esta lección trata de explicar cómo identificar los } \\
\text { riesgos a los que nos exponemos y cuantificarlos }\end{array}$ \\
\hline $\begin{array}{l}\text { Objetivos de } \\
\text { aprendizaje }\end{array}$ & $\begin{array}{l}\text { Conocer los principales riesgos a los que se está } \\
\text { expuesto habitualmente }\end{array}$ \\
\hline
\end{tabular}

Tabla 2. Descripción de la lección “¿Podemos cuantificar el riesgo?”

de cuantificarlas, razonada y razonablemente. Como materiales o recursos se recomienda al usuario la lectura de las fichas técnicas sobre: dinero, riesgo, presupuesto y flujo de caja. Las actividades y herramientas de enseñanza propuestas son tres:

- Trabajo 1: Principales riesgos de la vida cotidiana (para identificar qué riesgos se suelen asumir en las decisiones de compra cotidianas).

http://www.dolceta.eu/espana/Mod7/Actividad-Losprincipales-riesgos.html

- Trabajo 2: Cuantificando el riesgo (para determinar la probabilidad de ocurrencia de circunstancias que pueden desembocar en situaciones de riesgo, y valorar la conveniencia de asumirlo y las medidas a aplicar para reducirlo). http://www.dolceta.eu/espana/Mod7/Actividad-Cuantificando-el-riesgo.html

- Quiz 1: Principales riesgos a los que nos enfrentamos (cuestionario que permite comprobar los conocimientos adquiridos). Las pruebas están disponibles online para que puedan ser consultadas/resueltas en tiempo real, y también es posible descargarlos en formato pdf para facilitar su posterior resolución, en caso de que los estudiantes del aula no dispongan de acceso a internet.

http://www.dolceta.eu/espana/Mod7/Cuestionario-Principales-riesgos-a.html

El sistema de evaluación propuesto se basa en la participación en una discusión en clase y en los resultados de las tareas realizadas.

Adicionalmente se facilitan referencias bibliográficas y recursos web, así como actividades complementarias. Por ejemplo, en la lección "¿Podemos cuantificar el riesgo?" se propone hacer un seguimiento, durante un período determinado, de la prensa diaria para analizar sucesos ocurridos que hayan implicado una situación de riesgo, y analizar cómo se podría haber actuado para disminuirlo.

\section{Objetivo / Resultados de Aprendizaje / Objetivos del profesor}

Tiempo

Competencias

Materiales, recursos, contenidos, información de entrada, enlace a hojas de cálculo de Servicios financieros o Módulo de servicios financieros, etc.

Actividades, herramientas (con enlace a la herramienta)

Paso a paso del proceso

Icono de la prueba (enlace a quiz)

Evaluación de la prueba

Actividades posteriores a la lección

Bibliografía, lecturas, enlaces a notas, otros módulos de Dolceta,

otras webs, bases de datos de la Comisión Europea, etc.

Tabla 3. Ejemplo de la estructura de una lección

\section{Glosario y fichas técnicas}

Para completar los contenidos el panel de expertos europeos redactó fichas técnicas comunes para cada país. Son descripciones exhaustivas sobre un tema concreto. No todos los países las han incluido. España, por ejemplo, no se incluyó la ficha sobre banca islámica, de baja introducción hoy en día.

Estas fichas son útiles porque ofrecen información teórica y práctica sobre temas que no están cubiertos por el módulo 2 de servicios financieros de Dolceta, módulo diferente, aunque relacionado con el de alfabetización financiera. En él se trata gestión del presupuesto familiar, crédito al consumo, crédito hipotecario, cuenta corriente, medios de pago e inversiones. En cambio, en el módulo 7, que es el módulo de alfabetización financiera que presentamos aquí, se incluyen otros aspectos como finanzas sociales, banca ética, seguros, inflación, riesgo, reclamaciones sobre consumo, apertura de un negocio, bolsa, historia del dinero, planificación económica de un viaje, etc.

En segundo lugar, su contenido ofrece un breve desarrollo teórico de temas financieros e incluye enlaces de interés.

Además las fichas completan la información de la lección y están escritas de forma sencilla y adecuada a la edad y al perfil de los estudiantes destinatarios.

Por último, para España se elaboró un glosario con un total de 41 términos financieros, como por ejemplo, acciones, código de barras, bonos, flujos de efectivo, estabilidad de precios, desempleo, etc.

\subsection{Cuestionarios y hojas de trabajo}

En las experiencias de e-learning es importante proporcionar información a los participantes para que puedan evaluar y realizar un seguimiento sobre la evolución de su trabajo y aprendizaje autónomo (Benito; Cruz, 2005). Por ello se indicó a los expertos nacionales que prepararon los materiales del módulo que debían confeccionar, al menos, un cuestionario (quiz) en cada lección para que los usuarios de Dolceta autoevaluaran los conocimientos adquiridos.

Se diseñaron 3 tipos de cuestionarios: "Bien o mal", "rellenar espacios en blanco", y "múltiples opciones". Este último debía contener como mínimo 10 preguntas con al menos 
tres alternativas, siendo sólo una correcta. En todas las respuestas (correctas e incorrectas) se incluía un comentario aclaratorio adaptado al nivel del estudiante. Para hacer las preguntas del quiz más atractivas se obligó a insertar alguna imagen (libre de derechos) en cada una.

Además, en cada lección se ha propuesto al menos una hoja de trabajo o actividad a realizar por el estudiante o usuario. Por ejemplo, cuantificar la inversión inicial de un negocio, saber cómo y cuándo poner una reclamación, saber cómo solicitar un crédito, etc.

Todas las actividades fueron diseñadas para que los estudiantes/usuarios pudieran responderlas online, o bien, en el caso de que no dispusieran de conexión a internet, que pudieran hacerlo contestando el correspondiente documento en formato pdf que debían haberse descargado previamente. El total de actividades o herramientas diseñadas, tanto quizzes como hojas de trabajo, fueron 24 para primaria, 22 para secundaria y 22 para alumnos de bachillerato.

\section{Organización y desarrollo del módulo de alfabetización financiera}

El módulo fue propuesto de manera que su esquema básico de contenido y estructura pudiera ser utilizado por todos los países participantes en el proyecto, sin importar su identidad cultural. La base y el esquema del módulo son idénticos para todos los países pero adaptados a las necesidades formativas en materia financiera propias de los estudiantes en el contexto de su país.

El esquema básico de contenidos realizado por la DG Sanco, incluye:

- Préstamos a estudiantes.

- Actividades online de las entidades financieras.

- Cooperativas de crédito.

- Ahorro a través de fondos de inversión.

- Seguros.

- Conceptos básicos y definiciones del dinero (historia, ahorros, deuda, etc.).

- Acciones a emprender si los servicios financieros no funcionan adecuadamente (cómo y a quién reclamar, función del regulador, etc.).

\subsection{Diseño}

Se llevó a cabo entre abril de 2008 y enero de 2010. Fue nombrado un panel de expertos -con representantes de la CE y miembros del equipo técnico y de gestión de Dolceta- que definió los objetivos y diseñó la metodología y los contenidos a incluir: temas a elaborar por todos los países, estructura de las lecciones y documentación de apoyo a incluir en cada tema.

Se planificó en 3 fases:

\section{- Fase 1. Bases (entre 6-8 meses)}

a) Diseño del esquema, conceptos y contenidos principales del módulo por parte de un equipo internacional compuesto por especialistas en enseñanza y finanzas, junto con representantes de la $C E$ y el equipo técnico de Eucem y Dolceta. En una serie de reuniones en Bruselas se decidió trabajar en el plan de acción siguiente:
- Estructura de las lecciones, desde el punto de vista de buscar la mejor forma de aprendizaje.

- Lista de temas a tratar, de tal manera que se decidió crear las 4 secciones del módulo.

- Notas técnicas de apoyo a preparar por un equipo seleccionado por Eucem: banca online, dinero, presupuesto y flujo de caja, pago con tarjeta, finanzas sociales, inflación, fondos de inversión, préstamos para estudiantes, riesgo, seguros, hacer una reclamación, banca islámica (en el Reino Unido).

b) Consulta con los equipos nacionales y los representantes de la CE sobre el diseño de los materiales propuestos.

c) Aprobación de la versión final.

d) Realización del marco técnico para incorporar los contenidos nacionales.

Continuando con los pasos b), c) y d), se llevaron a cabo nuevas reuniones en Bruselas a las que asistieron 81 representantes de 25 países, durante las cuales se realizaron 4 sesiones paralelas de trabajo. Asimismo, hubo una sesión plenaria para la aprobación final de las principales líneas del proyecto. Los grupos de trabajo se estructuraron matricialmente: los contenidos técnicos, por un lado, y los recursos educativos, por otro.

\section{- Fase 2. Implementación de la versión completa (1 año)}

En el idioma de cada país y adaptado a las normativas nacionales y a su cultura.

El equipo de diseño de expertos de cada país realizó su propia lista detallada de los temas, contenidos y ejercicios. En España, esta adaptación fue realizada por un equipo dirigido por la Universidad Rovira i Virgili (URV). Este desarrollo se centró específicamente en los siguientes aspectos:

a) La estructura de los equipos en cada país fue una decisión del mismo. En general, el equipo estaba compuesto por un experto pedagógico, un experto del módulo a desarrollar, y un director técnico. En el caso español, dos expertos desarrollaron el módulo. Estos equipos recibieron indicaciones de contenido y estilo de redacción para los materiales de cada módulo (lecciones, notas técnicas, glosario, cuestionarios, palabras, imágenes a insertar, etc.).

El módulo debía incluir los recursos educativos, guión de las lecciones, enlaces web, concursos, juegos e ideas adjuntando sugerencias para los profesores acerca de cómo se podía utilizar dicho material en el aula. En concreto, el guión de cada lección debía indicar el tiempo necesario para hacerlo, competencias desarrolladas en el módulo, los objetivos de aprendizaje y herramientas adicionales que el profesor precisaba para su impartición.

El material debía estar planificado para 2-3 sesiones de 60 minutos de trabajo $y$, aproximadamente, 30 minutos de trabajo para los profesores; incluyendo el tiempo para desarrollar las clases, revisar el material (contenidos, recursos adicionales y fuentes de información), evaluar y realizar el seguimiento posterior de los estudiantes.

b) El equipo técnico de Eucen procedía a supervisar la correcta planificación del proyecto siguiendo el calendario establecido. Adicionalmente, dichos técnicos comprobaban 
detalladamente cuestiones formales de las pruebas y quizzes; verificando la existencia de explicación en las respuestas facilitadas, gráficos adecuados y figuras e ilustraciones acordes con las edades a las que se destinaban las distintas pruebas.

\section{- Fase 3. Corrección y comprobación técnica del funciona- miento (6 meses)}

La revisión incluyó cuestionarios, enlaces a los archivos adjuntos, visualización de imágenes y homogeneidad de las lecciones y los ejercicios propuestos. Esta fase fue realizada primero por los equipos nacionales y después por técnicos de Dolceta.

\subsection{Control de calidad}

La evaluación de la calidad de la web puede medirse a través de la percepción de los usuarios. Sin embargo, en una primera etapa, también puede garantizarse mediante la evaluación o revisión de los materiales por parte de expertos ajenos a los equipos que los elaboraron. Siguiendo este enfoque, para controlar la calidad del módulo se llevaron a cabo los siguientes pasos:

- Un experto pedagógico de cada nación y un experto del equipo Eucen/Dolceta han revisado el contenido y comprobado que los contenidos son adecuados a los objetivos de Dolceta y, además, se han adaptado al nivel de edad propuesto.

- Expertos nacionales y expertos del equipo Eucen/Dolceta han revisado técnicamente los materiales elaborados por cada país, comprobando que todos los equipos han seguido enfoques similares.

Los equipos nacionales recibían comentarios y recomendaciones para modificar el material tras cada revisión y, posteriormente estos equipos eran controlados periódicamente. De hecho, la etapa final (fase 3), supone la corrección derivada del proceso de evaluación y control. Finalmente, el equipo técnico de Dolceta se encargó de la construcción de la web y de asegurar su correcto funcionamiento.

\section{Conclusiones}

Se ha descrito cómo se construyó el módulo de Dolceta sobre alfabetización financiera en el caso de la plataforma española: contenido y estructura y cómo se organizó el trabajo entre los distintos países europeos que lo han desarrollado.

Este artículo ofrece dos contribuciones relevantes. La primera es describir la organización de esta experiencia de e-learning, para darla a conocer y orientar otras iniciativas similares. Aunque este proyecto formativo se ha llevado a cabo a nivel europeo, el trabajo realizado desde España permite ser utilizado en otros países y temáticas. La organización del módulo es el resultado de numerosas mejoras detectadas en la elaboración de los módulos anteriores. Puede decirse, por tanto, que el trabajo presentado podría ser una referencia para otras experiencias educativas online en materia financiera.

La segunda contribución es remarcar la importancia de promover la alfabetización financiera, y la relevancia de que este esfuerzo se haga desde edades tempranas. Por ello
Dolceta proporciona a los profesores herramientas y materiales para sus estudiantes al inicio de su carrera educativa, tratando de aumentar la calidad y homogeneidad de la alfabetización financiera.

Con la descripción de este módulo online se ha puesto de relieve la utilidad de esta experiencia educativa para promover un consumo responsable en el marco de la Comunidad Europea. Sus contenidos son básicos pero útiles para las decisiones diarias de los consumidores en cuestiones financieras. Este aspecto se vuelve más relevante si pensamos que a menudo la gente se sorprende de lo rápido que las prácticas y los instrumentos financieros evolucionan en el contexto actual, y que pueden aprovechar una herramienta formativa gratuita y sencilla de utilizar, que permite reciclar sus conocimientos en materia financiera y de consumo.

Dolceta ha sido diseñado para un mercado global, que integra múltiples países y contextos. Esto implica que sus materiales estén disponibles en distintos idiomas, y que proporciona una formación uniforme para el conjunto de la UE, con temas financieros similares y notas técnicas idénticas para todos los países participantes, aunque incorporando también ciertas particularidades y características de cada país.

Dolceta fue inaugurado oficialmente en junio de 2006 y el módulo de alfabetización financiera comenzó a estar accesible en 2009. Ello no permite aún verificar su implantación ni evaluar su eficiencia, por lo que el objetivo de este trabajo ha sido básicamente descriptivo. De hecho, se puede considerar que el proyecto no ha hecho más que comenzar. Después de su puesta en marcha se está difundiendo entre los centros formativos interesados, y con presentaciones en encuentros y congresos, pero queda todavía un gran camino por recorrer para conseguir un amplio conocimiento entre la población. A medida que vaya siendo utilizado se dispondrá de más información sobre la satisfacción y valoración de sus usuarios, lo que constituirá objeto de una futura investigación.

\section{Bibliografía}

Alrø, Helle; Skovsmose, Ole; Skanstrøm, Mikael. Student inquiry co-operation in mathematics education. Auchland, Nueva Zelanda: Mathematics Education Unit, Department of Mathematics, University of Auchland, 2000.

Arvanitis, Spyridon. "E-Learning programs as royalty investments for financial corporations". British journal of educational technology, 2010, v. 41, n. 5, pp. E70-E74. http://dx.doi.org/10.1111/j.1467-8535.2009.00971.x

Bailey, Sue; Kitson, Michael. Guidelines for consumer education for adults. Viena: Consumer Education for Adults Network, 2006.

Barker, Philip. "On being an online tutor". Innovations in education and teaching international, 2002, v. 39, n. 1, pp. 3-13.

http://dx.doi.org/10.1080/13558000110097082

Benito, Agueda; Cruz, Ana. Nuevas claves para la docencia universitaria en el Espacio Europeo de Educación Superior. Madrid: Narcea Editores, 2005. ISBN: 9788427715013 
Benito, Diana. "Aprendizaje en el entorno del e-learning: estrategias y figura del e-moderador". Revista de universidad y sociedad del conocimiento, 2009, v. 6, n. 2, pp.1-8.

http://www.uoc.edu/ojs/index.php/rusc/article/viewArti cle/v6n2-benito

Cabero, Julio; Gisbert, Mercè. La formación en Internet: guía para el diseño de materiales formativos. Sevilla: MAD, 2005. ISBN: 9788466520546

Carnell, Eileen; Lodge, Caroline. Supporting effective learning. London: Paul Chapman, 2002, ISBN: 0761970460

Gottschau, Jette; Nielsen, Sanne-Schnell. "Cross-disciplinary consumer citizenship education". En: Tangen, Dag; Thoresen, Victoria (eds.). Taking responsibility CCN conference proceedings. Bratislava: Consumer Citizenship Network, 2005, pp. 141-149. ISBN: 8276714951

Ma, Louis; Vogel, Doug; Wagner, Christian. "Will virtual education initiatives succeed?" Information technology and management, 2000, v. 1, pp. 209-227.

http://isnet.org.cn/xxjsjy/webcourse/course/ztyx/read/ chapter5/Will\%20virtual\%20education\%20initiatives\%20su cceed.pdf

http://dx.doi.org/10.1023/A:1019177109389

McGill, Tanya; Klobas, Jane. "A task-technology fit view of learning management system impact". Computers \& education, 2009, n. 52, pp. 496-508.

http://dx.doi.org/10.1016/j.compedu.2008.10.002

Ministerio de Educación, Cultura y Deporte. Documento marco: La integración del sistema universitario español en el
Espacio Europeo de Enseñanza Superior. Madrid: Ministerio de Educación, Cultura y Deporte, 2003.

http://www.dolceta.eu/espana

http://www.uhu.es/convergencia_europea/documentos/ documentos-2007/docmarco_MEC_feb2003.pdf

Ministros europeos de educación. Declaración de Bolonia, 1999.

http://www.bologna-bergen2005.no/Docs/00-Main_doc/ 990719BOLOGNA_DECLARATION.PDF

Robinson, Julian. "Developing a screen-capture reusable learning object for undergraduates". En: $7^{\text {th }}$ European conf on e-learning, 2008, v. 2, pp. 366-375.

Stoll, Louise; Fink, Dean; Earl, Lorna. Sobre el aprender y el tiempo que requiere. Barcelona: Octaedro, 2004, ISBN: 8480636300

Sun, Pei-Chen; Tsai, Ray J.; Finger, Glenn; Chen, Yueh-Yang; Yeh, Dowming. "What drives a successful e-learning? An empirical investigation of the critical factors influencing learner satisfaction". Computers \& education, 2008, v. 50, n. 4, pp. 183-202.

http://ebiz.bm.nsysu.edu.tw/2009/m954011064/ References/20081129Sun2006.pdf

http://dx.doi.org/10.1016/j.compedu.2006.11.007

Yamashita, Yasuo; Takahashi, Hiroshi; Terano, Takao. “Approach to learning financial theory through business gaming". En: Proceedings of SICE annual conference, 2008, v. 1, n. 7, pp. 2.573-2.577.

http://dx.doi.org/10.1109/SICE.2008.4655120

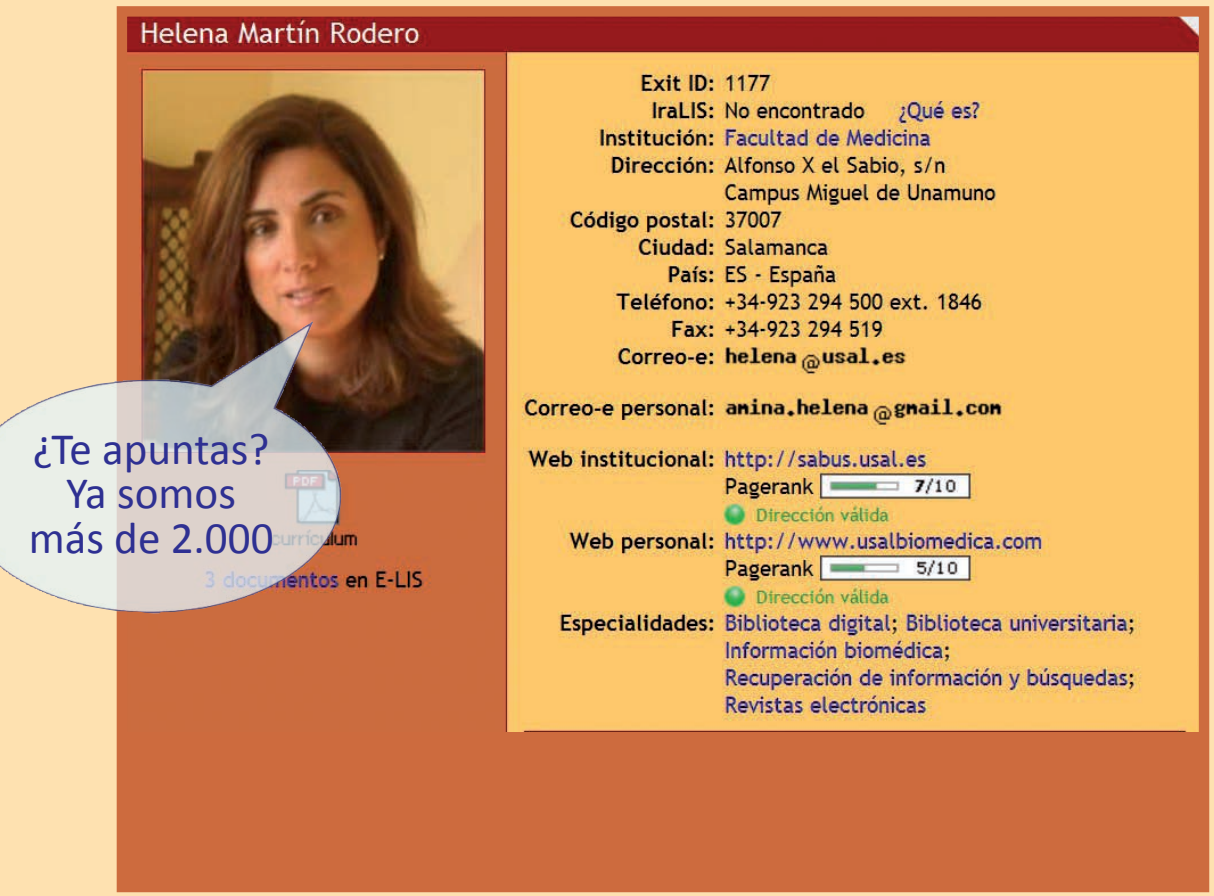

http://directorioexit.info
Para titulados con más de 1 año de experiencia, que hayan publicado algún artículo o ponencia o puedan dar clase más de 1 hora. 